Davies, R.D., Pedlar, A. and Mirabe1, F., 1977. Mon. Not. R. astr. Soc., in press.

Sunyaev, R.A. and Zeldovich, Ya. B., 1972. Astr. Astrophys., 20, 189;

1975. Mon. Not. R. astr. Soc., 171, 375.

\title{
DISCUSSION
}

Sunyaev: This is a very important observation, because this is a possible way to observe protoclusters of galaxies at the stage when there were no galaxies and there was only gas. The mass of cold gas may be 10 to 100 times greater than today, because the gas was not condensed into stars at that time. I want to mention that the limits obtained by Dr Davies and his colleagues are very useful and give an upper limit on the lifetime of a protocluster at this stage. They are also important when we choose the epoch of cluster formation. I must mention also that in the open Universe $(\Omega<1)$ it is much more difficult to find these objects because their angular dimensions are smaller. Therefore the restrictions on $M, V$ and $\Delta t$ depend strongly on the accepted value of $\Omega$. We are very grateful to Dr Davies for obtaining data so important for our theory.

There is an interesting possibility of finding the redshifts of distant radio sources at radio wavelengths. If there are protoclusters or galaxies or clouds of cold matter on the line of sight between the source and the observer, absorption radiolines might appear in the radio spectrum. They must be redshifted. For example, the detection of an absorption line in the metre waveband might be interpreted as a strongly redshifted $21(1+z) \mathrm{cm}$ line. In this case we can measure the redshift of absorbing matter and find a lower limit to the redshift of the source. It is important that there are no we11-known radio lines in the metre waveband.

It is possible also to predict other strong absorption lines in the spectra of distant radio sources. Among these are $\lambda 2.6 \mathrm{~mm}$ CO line (redshifted to the $1 \mathrm{~cm}$ band) and $\lambda 6 \mathrm{~cm}$ line of formaldehyde (redshifted to the decimetre waveband).

\section{ORIENTATION OF SPIRAL GALAXIES AS A TEST OF THEORIES OF GALAXY FORMATION}

\section{J. Jaaniste and E. Saar}

The cosmological turbulence theory and the theory of adiabatic fluctuations predict different orientations of galaxies in clusters and superclusters. The first theory favours the alignment of the planes of galaxies with the supergalactic plane, whereas the planes of spiral galaxies formed according to the second theory are perpendicular to the plane of a supergalaxy.

We compared these alternative predictions with the observed distri- 
bution of orientations of spiral galaxies in the Local Supercluster within the volume of a radius of $50 \mathrm{Mpc}$ (Jaaniste and Saar 1977). As a result, the picture expected in the turbulence theory (alignment of the planes) is excluded at a high confidence level. This is due to the observed concentration of face-on galaxies towards the supergalactic equator, a fact that cannot be explained by the turbulence theory. As only nearly edge-on galaxies have been used in most statistical studies of orientation, the effect of face-on galaxies has been ignored so far.

The distribution of poles of spiral galaxies is significantly concentrated towards the supergalactic plane in agreement with the theory of adiabatic fluctuations.

\section{REFERENCE}

Jaaniste, J. and Saar, E., 1977. Tartu Astrophys. Obs. Preprint A-2.

\section{DISCUSSION}

de Vaucouleurs: Do you conclude that there is an excess of galactic planes parallel or perpendicular to the supergalactic planes?

Saar: There is a definite excess of galaxies with planes perpendicular to the supergalactic plane.

de Vaucouleurs: Do your results agree with those of Roberts and Reinhardt or not?

Saar: No, they disagree, and this is probably due to the difference of our samples. Roberts and Reinhardt used all the galaxies of the Reference Catalogue, while we considered only those galaxies that undoubtedly belong to the Local Supercluster.

Reinhardt: You are aware that your investigation contradicts the result of Reinhardt and Roberts, Nilson and de Vaucouleurs, all derived from slightly different data samples and giving a weak parallelism of planes of galaxies to the equatorial plane of the Local Supercluster. Of course, your sample is smaller and different from all of those used in the previous investigations. Also your method is different from those of your precursors. Thus without reanalyzing your data with another method, it is difficult to say how your results compare to the previous ones. 